\title{
Cad Software Based Design of Yarn Guide Profile
}

\author{
Mrs. Pragnya S.Kanade ${ }^{1}$, Dr. (Prof) S.S.Bhattacharya ${ }^{2}$, Mr. Akash Pandey ${ }^{3}$ \\ 1,2(Textile Engineering Dept., Faculty of Tech. and Engg., M.S.University of Baroda, Gujarat, India) \\ 3 (Mechanical Engineering Dept., Faculty of Tech. and Engg., M.S. University of Baroda, Gujarat, India )
}

\begin{abstract}
The traversing mechanism on a winding machine causes the yarn guide and hence the yarn to reciprocate. It was thought that a very interesting work would result if this reciprocating motion could be eliminated. This work is an attempt to eliminate the too and fro motion of the yarn guide wherein the guide profile ensures proper transfer of yarn with the desired pattern and pitch. The change in yarn direction after completing one axial traverse poses the most challenging aspect in designing an efficient guide profile. With idealizing assumption of the yarn behaving as a thin wire under the taut condition between three support points at the top ring, profile point on the guide and the bobbin, the ideal profile for consistent guidance of the yarn has been developed.
\end{abstract}

Keywords: chain, transfer-zone, tension, $\quad$ yarn-guides.

\section{INTRODUCTION}

On a winding machine the yarn needs to be traversed too and fro, which is most commonly done either by a cam or a grooved drum. But this work was taken up with the intention of developing an alternative method to traverse the yarn, for which here, an endless chain has been used. The patent ${ }^{1}$ has been taken as the reference which makes use of profiled guide. Here with help of software Pro Engineer 4.0, the position of guide in the transfer zone has been simulated and accordingly the correct profiles of the giver and taker guide have been developed so that not only the traversing but even the transfer takes place effectively. Thus the most important requirement for this system to work was to design a yarn carrier (guide) which not only takes the yarn from one end of the traverse to the opposite end but also efficiently transfers it from the top yarn carrier to the bottom one and vice versa.

\section{YARN GUIDE REQUIREMENTS}

As mentioned earlier an endless chain due to its positive nature, has been used on which the yarn guide is mounted. The arrangement finalized for achieving traverse and transfer is shown in Fig. 1.

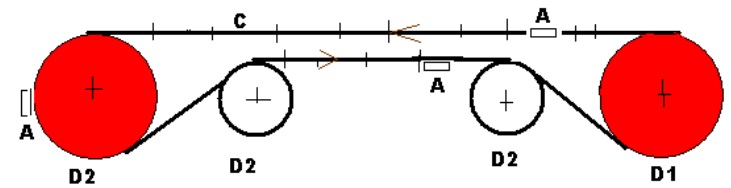

Fig. 1

A: guiding element (three of same type), D1: driving sprocket, D2: carrier/driven sprocket, C: chain

The driver sprocket (D1) drives the chain. The top chain, the guide and hence the yarn travels towards the left in the figure, and vice versa. Since the chain does not reverse when the guide reaches its extreme end of its traverse, problems due to guide reversal are avoided. The other sprockets namely the one at the extreme and the other two sprockets in the bottom line are carrier /driven sprockets have been named (D2). A represents the guide that have been placed at equal intervals from each other. So the entire traverse can be divided into three main divisions namely, the two extremes and the central. The two extreme zones can be called as the transfer zone whereas the central can be called as the traverse zone. The transfer zones have been very critical and the profiles have been designed as per the geometry in that region. The arrangement is such that when the top chain enters the transfer zone another yarn guide mounted on the bottom chain also enters the transfer zone on the lower side. In this zone the yarn should get transferred from the top yarn the bottom one and the point at which this occurs is the transfer point. After the transfer, the yarn is now in the guide on the bottom chain which is moving towards the right, thus causing reversal of the yarn. This system does not contain any reciprocating element, which is an added advantage. Now with the basic set-up ready, the next important challenge was to design a yarn guide which would give a smooth yarn transfer when the yarn reached the transfer zone. 


\section{MATERIALS}

On the commercial winding machines, to traverse yarn only one guide is used since it is of reciprocating type. So the guide is normally a piece which has a hole at center lined with ceramic, through which the yarn passes. This shape would not be suitable for the above application since the yarn has to be moved out of one of the guides and moved into the other one. The following considerations would have to be made before the exact profile of the guide can be designed:

a. The yarn guide would have to be open from its front side.

b. The guide would have to be anchored to the reciprocating element (chain).

c. At the transfer point the yarn needed to be removed from top guide and made to sit in the bottom guide or vice versa. So it was imperative to device a mechanism whereby the yarn could be gradually ejected from one of the guides and smoothly transferred to the other one.

There were two options, either to make use of a profiled guide which would do two fold function that is firstly to carry the yarn too and fro and secondly its profile would cause the yarn to be ejected from the giver guide at the same time help the receiver guide to receive the yarn, and can be called self acting. For this purpose survey was done and patents ${ }^{1,2,3 \& 4}$ were searched. The patent ${ }^{1}$ shows profiled guide which enables transfer to take place at end of each traverse. It makes use of chain as the medium to carry the yarn. It also includes modification on the original profile. Patent ${ }^{2}$ is also related to a profiled guide mounted on chain. It is claimed that the system has been specially developed for the yarn on solidification from the melt spinning process. There are at least two guiding members who take yarn too and fro. This mechanism also used co-axial sprockets and chains due to which reversal of the traversing mechanism does not occur. Patent ${ }^{3}$ is related to the guide profile for rotating wings. The patent ${ }^{4}$ is related to belt traverse. In this system an endless belt, each of which carries yarn traversing fingers arranged to move in the opposite directions to traverse the yarn axially of a winding package are used. Auxiliary yarn engaging members are positioned to facilitate reversal of the yarn at the package ends. The second option would be to make use of a yarn guide and a separate tapered profile. The tapered profile would be helpful to bring the yarn gradually out of the giver guide and transfer it to the taker guide. While finalizing the guide designs various shapes were thought of, some of which have been shown below in Fig.2.
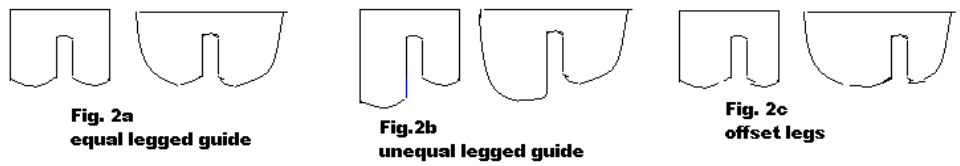

Fig. 2

It was finally realized that number of such profiles could be devised. Hence it was decided to take help of the software (Pro Engineer 4.0) available to design the yarn guide and the profile by actually simulating the situation as it existed on the machine.

\section{METHODS}

The traversing assembly was modeled to scale as shown in Fig. 3. The blue color line indicates the yarn, which is coming from the supply package mounted at the back of the machine. Just before the yarn enters the traversing guide it passes through a stationery yarn guide. The position of this guide is offset from the yarn guide axis and is 10 " above the machine frame which supports the sprockets.

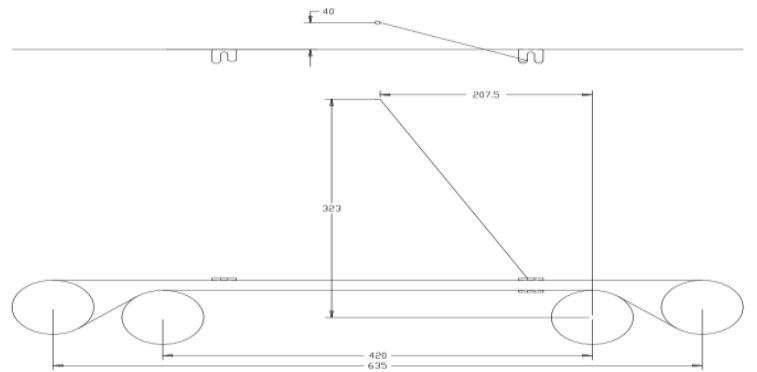

Fig. 3 Model of traversing assembly

While carrying out this work, the total transfer zone distance had been divided into equal distances with the assumption that the yarn will be guided out of the trailing edge of the bottom guide to be taken into the leading edge of the top guide. The guides are placed at the beginning (inside the groove of the giver guide) and end (edge of the taker guide) points of transfer zone as indicated in Fig. 4. The yarn, modeled in form of a wire of same diameter as the yarn is also modeled at the initial and final position of the transfer zone. Guides were 
located at each of these interval points decided. The yarn should be pushed out from the semi-circular edge of the bottom guide. This is achieved by using a stationery profile indicated in red color. By this time the yarn would become uncontrolled and would try to come to the center due to the tension, if the upper guide does not take control of the yarn. To ensure this a stationery guide profile in pink color is shown and is made to overlap the red curve. This profile of the upper stationary guide (pink) starts to push the yarn inside the groove of the top guide.

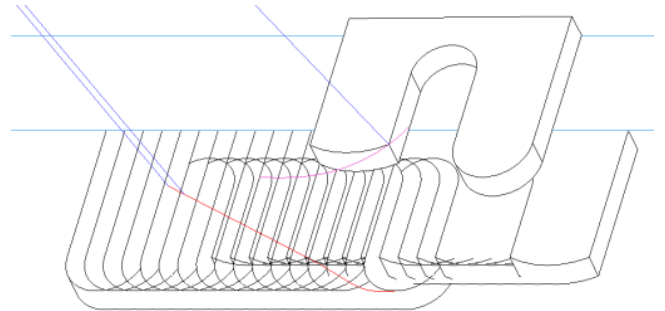

Fig. 4 Successive Guide Locations in Transfer zone at the beginning.

Moving from left to right, the Fig. 5 is reached which indicates the end of transfer. So in short it can be said that the yarn at the start of the transfer would be pushed out of the lower guide due to the stationery red profile. But the task of pushing the yarn inside the groove of the top guide is done by the pink stationery profile. When this top guide reaches the transfer zone on the left side the same procedure will be followed only this time the yarn will be pushed out from the top guide and into the bottom guide.

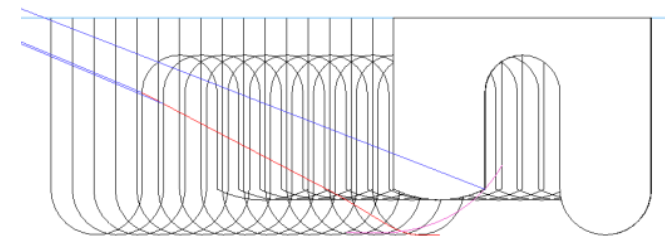

Fig. 5_Profiles of Stationary Guides at end of transfer

The points on the red profile, representing the bottom stationary guide, are important from the point of view of distance covered during each interval in order to maintain stability of the yarn. Hence, points are generated on the path to fit a curve. While for the upper stationary guide, maintaining an overlap with the red curve a cubic profile is fitted to guide the yarn into the groove of the top guide.

The Figs. $6 \mathrm{a}, 6 \mathrm{~b}$ and $6 \mathrm{c}$ show the position of bottom guide at the beginning, intermediate and at the end of the transfer.

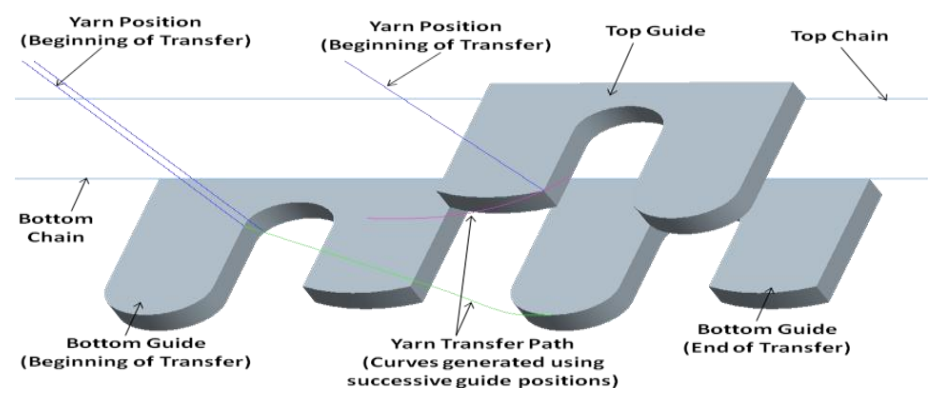

Fig. 6a shows the extreme left position of the guides in the transfer zone (beginning of the transfer)

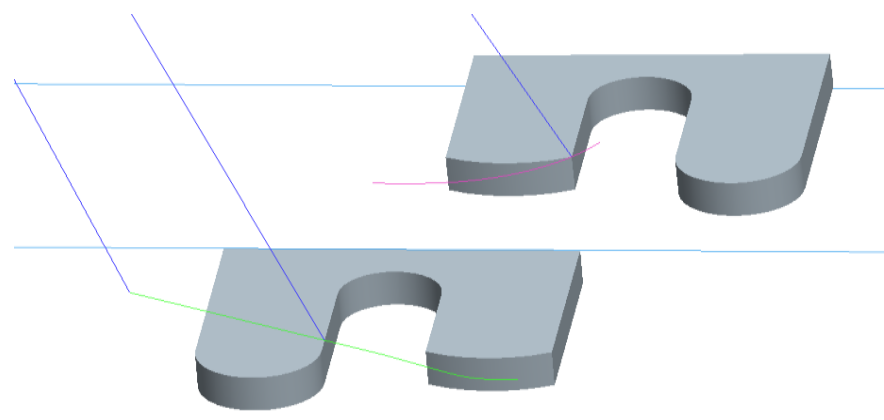

Fig. $6 \mathrm{~b}$ shows the intermediate position of the top and bottom guide in transfer zone 


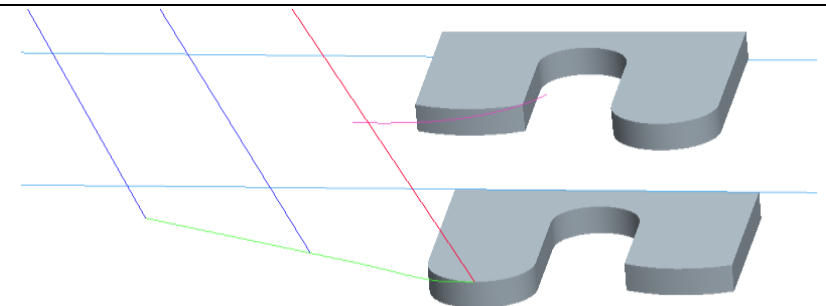

Fig. $6 \mathrm{c}$ shows the extreme right position of the guides in the transfer zone

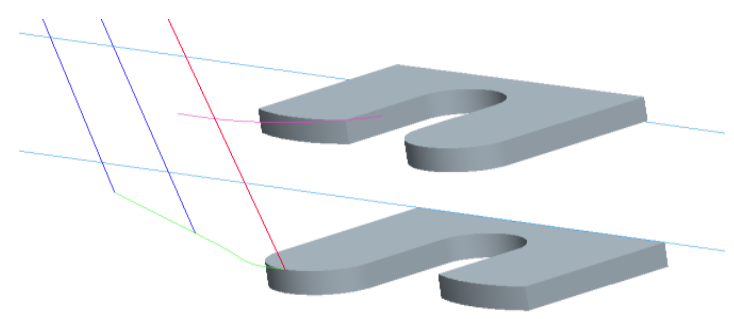

Fig. $6 \mathrm{~d}$ is the same position as Fig. 4 only shown at a different angle

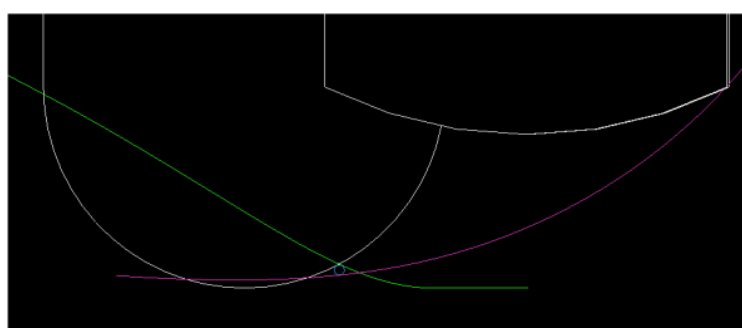

Fig. 6e is the top view of the two guides along with the profiles and yarn at the end of the transfer.

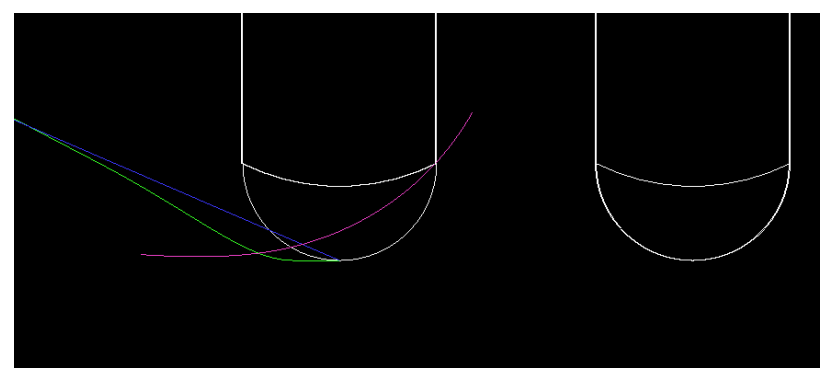

Fig. $6 \mathrm{f}$ shows the position when the guides are exactly one below the other

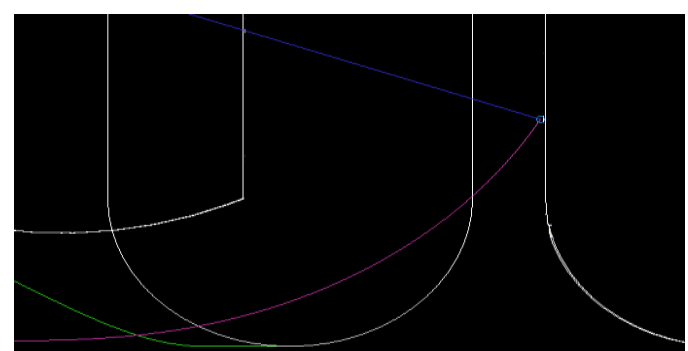

Fig. 6g shows the position when complete transfer has taken place

The yarn has safely been deposited into the ' $U$ ' of the top guide. Based on the profiles obtained in this form, the bottom and top stationary guides are developed as shown in Fig. 7 and Fig. 8 respectively.

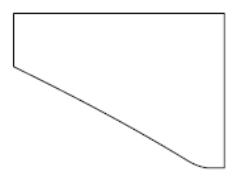

Fig. 7 Top stationery guide

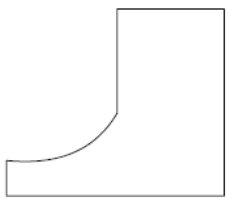

Fig. 8 Bottom stationery guide 
The yarn on one side is being guided by the green profile to be pushed outside the lower guide profile whereas the pink guide at the top is guiding it to be pushed inside top guide. Yarn in this position has come in the interference of both the guides.

The positions of the top and bottom guides on the machine to scale are indicated in red in Fig. 9 with respect to the bottom and top guides.

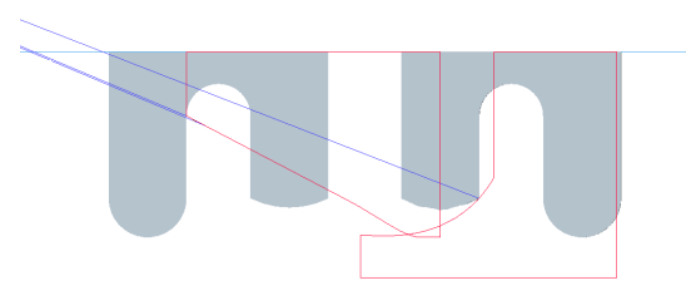

Fig. 9 Location of Bottom and Top Stationary Guide

\section{RESULTS AND DISCUSSIONS}

The scheme with two moving guides poses the problem of uncontrolled yarn during the end of the traverse at the left and right extreme positions. This leads to inconsistency in winding on the bobbin. In order to control the yarn during the extreme positions of direction reversal, the option of providing stationary guides has been developed using ideal positioning of the yarn.

The case of transfer at the right hand side of the traverse is considered and the profile of the stationary guides is developed to move the yarn from the bottom guide into the top guide. The relative position of the guides on the machine is also developed to scale in order to have proper control on the yarn.

The guide profiles are developed considering that the yarn remains taut during transfer of control. The addition of stationary guides leads to additional support point for the yarn between the top stationary guide and the winding point on the bobbin and actually helps in keeping the yarn taut during transfer. However, on account of the elasticity and dynamic forces, the yarn will deflect. To predict the domain of yarn movement requires an in-depth study involving implementation of simulation techniques like monte-carlo simulation with development of a random-order model to find the envelope of movement of the yarn. A flexible profile can be developed and superimposed on the mean profile developed considering the yarn to be taut.

\section{CONCLUSION}

The profile so developed has been found to work for both the two options mentioned. With the guide and tapered profile arrangement, though the transfer success rate increased, the yarn came under lot of tension. In the transfer zone the yarn came under the influence of both the stationery profiles exerting pressure in the opposite directions. This problem could not be visualized at the time of simulating, since the work has been done considering the yarn to be a taut wire, which it is not thus causing surface damage to the yarn and stretching.

[1] Roland R.Nydegger - patent number US 2238128

[2] John Morris Horwood, Llanyravon, Cwmbran - patent number US3294327

[3] Naotoshi Otsuka, Mishia-shi, and Masayuki Nozawa, Numazushi - patent number US 3374961.

[4] Hans H. Richter, Cranston, R.I - patent number US 348935 